\title{
REMARKS ON A CONSTRUCTION OF DUISTERMAAT
}

\author{
ANTHONY SMALL*
}

\begin{abstract}
A construction of Duistermaat's, in which an auxiliary holomorphic curve in the line bundle $\mathscr{O}(4)$ on $P_{1}$ is associated to a minimal surface in $R^{3}$, is described in a new way and generalised to minimal surfaces in $\mathbf{R}^{n}$. If the minimal surface is complete and has finite total curvature then the auxiliary curve is algebraic.
\end{abstract}

\section{Introduction}

Suppose that $\phi: X \longrightarrow \mathrm{R}^{3}$ is a branched minimal immersion of a Riemann surface whose Gauss map to the unit 2 -sphere at the origin is non-constant. In [1], Duistermaat describes a lift of the Gauss map, away from its branch points, to a holomorphic line bundle; using some elementary representation theory this bundle is identified as being isomorphic to $\mathscr{O}(4)$ on $\mathrm{P}_{1}$. It is observed that there exists a canonical holomorphic 1-form on the total space of the bundle, the real part of which, when pulled back to $X$, integrates to return $\phi$.

The encoding of the geometry of a minimal surface into the auxiliary curve is explored. In particular, the branch points of the Gauss map, end structure, branch points in the metric and total Gaussian curvature.

It is shown that if $\phi$ is finitely branched and induces a complete metric of finite total Gaussian curvature then the auxiliary curve is part of an algebraic curve on the compact complex surface $\mathrm{P}(\mathscr{O}(4) \oplus \mathscr{O})$. A number of interesting examples are discussed.

Our purpose in this note is to give an alternative description of Duistermaat's lift which generalises easily to the study of maps to $\mathrm{R}^{n}$. It is shown, following [1], how to associate algebraic curves to finitely branched complete minimal immersions of finite total Gaussian curvature.

\footnotetext{
* The author is grateful to Hans Duistermaat for helpful remarks. He is indebted to the Mathematics Faculty of the University of Southampton, England, for its generous hospitality during the academic year 2001-2002, and thanks Victor Snaith for his invitation. He also thanks the Mathematics Section of the ICTP, Trieste, Italy, for its hospitality during February and March of 2002.
}

Received December 12, 2002. 


\section{Lifting the Gauss Map}

Let $Q_{n-2} \subset \mathrm{P}_{n-1}$ be the quadric hypersurface $\left(z_{1}^{2}+\cdots+z_{n}^{2}=0\right)$. Suppose that $X$ is a Riemann surface and $\phi: X \longrightarrow \mathrm{R}^{n}$ is a branched minimal immersion. The Gauss map $\gamma_{\phi}=[d \phi / d \xi]: X \longrightarrow Q_{n-2}$ is holomorphic; see [5] and [6] for further details.

Let $J$ denote the restriction of the universal bundle on $\mathrm{P}_{n-1}$ to $Q_{n-2}$ and $\pi_{2}$ be the map of $J$ induced by projection to the second factor of $\mathrm{P}_{n-1} \times \mathrm{C}^{n}$.

Definition 2.1. Let $E=T^{*} Q_{n-2} \otimes J$, and $\pi_{E}: E \longrightarrow Q_{n-2}$ be the projection map. There is a canonical $C^{n}$-valued holomorphic 1-form $\Omega$, on the total space of $E$ : for $w \in T_{q}^{\prime} E$, let $\Omega(w)=\pi_{2} \circ q \circ d \pi_{E}(w)$.

Now fix a smooth curve $\mathscr{G} \subset Q_{n-2}$.

Definition 2.2. Let $L \mathscr{G}=\left.T^{*} \mathscr{G} \otimes J\right|_{\mathscr{G}} \longrightarrow \mathscr{G}$, and $\pi_{L}: L \mathscr{G} \longrightarrow \mathscr{G}$ be the projection map. Observe that there is a canonical $\mathrm{C}^{n}$-valued holomorphic 1form $\Omega_{\mathscr{G}}$, on the total space of $L_{\mathscr{G}}$ : for $v \in T_{r}^{\prime} L \mathscr{G}$, let $\Omega_{\mathscr{G}}(w)=\pi_{2} \circ r \circ d \pi_{L}(v)$.

Given a branched minimal immersion $\phi: X \longrightarrow \mathrm{R}^{n}$, such that its Gauss map $\gamma_{\phi}$ is non-constant and takes values on $\mathscr{G} \subset Q_{n-2}$, we can define a lift of $\gamma_{\phi}$, away from $B_{\gamma_{\phi}}=\left\{\xi ; d \gamma_{\phi}(\xi)=0\right\}$, in the following way: for $\xi \in X \backslash B_{\phi}$, pick an inverse to $\gamma_{\phi}$ on a neighbourhood of $\gamma_{\phi}(\xi)$, which is such that $\gamma_{\phi}^{-1}\left(\gamma_{\phi}(\xi)\right)=\xi$.

$\Gamma_{\phi}: X \backslash B_{\gamma_{\phi}} \longrightarrow L \mathscr{G}$ is defined by: $\Gamma_{\phi}(\xi)=d\left(\phi \circ \gamma_{\phi}^{-1}\right)_{\gamma_{\phi}(\xi)}$.

Proposition 2.3. $\Gamma_{\phi}^{*} \Omega \mathscr{G}=\frac{d \phi}{d \xi} d \xi$.

Proof. For $v \in T_{\xi} X$,

$$
\begin{aligned}
\Omega_{\mathscr{G}}\left(d \Gamma_{\phi}(v)\right)=\pi_{2} \circ \Gamma_{\phi}(\xi) \circ d \pi_{L} \circ d \Gamma_{\phi}(v) & =\pi_{2} \circ \Gamma_{\phi}(\xi) \circ d \gamma_{\phi}(v) \\
& =\pi_{2} \circ d \phi(v) .
\end{aligned}
$$

Now, unless we start with a curve in $E$, there is not, for $n>3$, a canonical way to lift the Gauss map to $E$. However, $\Omega \mathscr{G}$ is related to $\Omega$ in the following way.

Write the inclusion $\iota: \mathscr{G} \longrightarrow Q_{n-2}$; this gives $\tilde{\imath}:\left.E\right|_{\mathscr{G}} \longrightarrow E$, which induces $\tilde{\iota}^{*} \Omega_{E}$ on $\left.E\right|_{\mathscr{G}}$. Let $\pi_{\mathscr{G}}:\left.E\right|_{\mathscr{G}} \longrightarrow \mathscr{G}$, denote the restriction of $\pi_{E}$ to $\left.E\right|_{\mathscr{G}}$. There is a natural map $\theta:\left.E\right|_{\mathscr{G}} \longrightarrow L \mathscr{G}$, namely $\theta(q)=q \circ d \iota$.

Proposition 2.4. On $\left.E\right|_{\mathscr{g}}: \tilde{\iota}^{*} \Omega_{E}=\theta^{*} \Omega$ g.

Proof. First observe that $\pi_{L} \circ \theta=\pi \mathscr{G}$ over $\left.E\right|_{\mathscr{G}}$, and hence

$$
d \pi_{L} \circ d \theta=d \pi \mathscr{G} .
$$


Next note that $\pi_{E} \circ \tilde{\imath}=\iota \circ \pi_{\mathscr{G}}$ and so

$$
d \pi_{E} \circ d \tilde{\iota}=d \iota \circ d \pi \varphi .
$$

For $w \in T_{q}^{\prime}\left(\left.E\right|_{\mathscr{G}}\right)$,

$$
\theta^{*} \Omega_{\mathscr{G}}(w)=\pi_{2} \circ \theta(q) \circ d \pi_{L} \circ d \theta(w) .
$$

Using the definition of $\theta(q)$ and (1) gives

$$
\theta^{*} \Omega_{\mathscr{G}}(w)=\pi_{2} \circ q \circ d \iota \circ d \pi_{\mathscr{G}}(w) .
$$

Applying (2) this gives

$$
\begin{aligned}
\theta^{*} \Omega \mathscr{G}(w) & =\pi_{2} \circ q \circ d \pi_{E} \circ d \tilde{\imath}(w) \\
& =\tilde{\iota}^{*} \Omega_{E}(w) .
\end{aligned}
$$

For $n=3$, the above gives Duistermaat's construction [1], described from the dual point of view. Note in particular that in this case, $E$ is isomorphic to the line bundle $\mathscr{O}(-4)$ on $\mathrm{P}_{1}$.

\section{Relationship with the Lie Correspondence}

Let Spé $\mathscr{O}(2)$ be the étalé space of $\mathscr{O}(2)$; classical osculation duality determines a canonical null holomorphic map $\Psi$ : Spé $\mathscr{O}(2) \longrightarrow \mathrm{H}^{0}\left(\mathrm{P}_{1}, \mathscr{O}(2)\right)$, given by: $\Psi\left([f]_{\zeta}\right)=\left(\frac{1}{2}\left(1-\zeta^{2}\right) f^{\prime \prime}(\zeta)+\zeta f^{\prime}(\zeta)-f(\zeta), \frac{1}{2} i\left(1+\zeta^{2}\right) f^{\prime \prime}(\zeta)-\right.$ $\left.i \zeta f^{\prime}(\zeta)+i f(\zeta), \zeta f^{\prime \prime}(\zeta)-f^{\prime}(\zeta)\right)$. Every non-planar minimal surface in $\mathrm{R}^{3}$ can be understood in terms of this map.

The correspondence was first found by Lie and rediscovered by Hitchin, see [7] for further details and references. In 2.6 of [1], Duistermaat asks after the relationship between the Lie correspondence and the construction of his paper; we describe it here. Analogous statements apply to $\mathscr{O}(n)$.

$$
\begin{aligned}
& \text { If } \omega=1 / \zeta \text { on } \mathrm{P}_{1} \text {, and } \tilde{f}(\omega)=-\omega^{2} f(1 / \omega) \text {, then } \tilde{f}^{\prime \prime \prime}(\omega)=\omega^{-4} f^{\prime \prime \prime}(1 / \omega) \text {. } \\
& \Omega\left([f]_{\zeta}\right):=\frac{d \Psi}{d \zeta} d \zeta=\frac{1}{2}\left(\left(1-\zeta^{2}\right), i\left(1+\zeta^{2}\right), 2 \zeta\right) f^{\prime \prime \prime}(\zeta) d \zeta
\end{aligned}
$$

is a canonical $\mathrm{H}^{0}\left(\mathrm{P}_{1}, \mathcal{O}(2)\right)$-valued holomorphic 1-form on Spé $\mathscr{O}(2)$.

Any null curve $\psi: X \longrightarrow \mathrm{H}^{0}\left(\mathrm{P}_{1}, \mathscr{O}(2)\right)$, whose Gauss map $\gamma_{\psi}$ is nonconstant, has a Gauss transform $\Gamma_{\psi}: X \longrightarrow \mathscr{O}(2)$; this lifts, away from the branch points of $\gamma_{\psi}$, to $\tilde{\Gamma}_{\psi}: X_{*} \longrightarrow$ Spé $\mathscr{O}(2)$, and $\left.\psi\right|_{X_{*}}=\Psi \circ \tilde{\Gamma}_{\psi}$, [7]. It is easy to show that

$$
\psi=\int \tilde{\Gamma}_{\psi}^{*} \Omega
$$




\section{Finite Total Curvature}

Suppose that $\phi: X \longrightarrow \mathrm{R}^{n}$ is a complete, finitely branched minimal immersion which induces a branched metric of finite total Gaussian curvature. Chern and Osserman showed that $X$ is conformally equivalent to $Y \backslash\left\{\xi_{1}, \ldots, \xi_{r}\right\}$, where $Y$ is compact and moreover the Gauss map of $\phi$ extends over the ends $\left\{\xi_{1}, \ldots, \xi_{r}\right\}$ to give a holomorphic map $\gamma_{\phi}: Y \longrightarrow Q_{n-2} . C(\phi)$, the total Gaussian curvature over $Y$, equals a constant times the homology degree of $\gamma_{\phi} ;$ see [5], [6].

Recall that the line bundle $L \mathscr{G}$ over $\mathscr{G}$ has a natural compactification to the ruled surface $\mathrm{P}\left(L_{\mathscr{G}} \oplus \mathcal{O}\right)$, in which a copy of $\mathscr{G}$, denoted $\mathscr{G}_{\infty}$, is added so that each fibre is completed to a copy of $P_{1}$, see [2]. The image of the zero section in $\mathrm{P}(L \mathscr{G} \oplus \mathcal{O})$, is denoted by $\mathscr{G}_{0}$. $C$ denotes a fibre of the bundle map $\mathrm{P}\left(L_{\mathscr{G}} \oplus \mathscr{O}\right) \longrightarrow \mathscr{G}$.

The following is a generalisation from the $n=3$ case of results in [1]: (i) follows immediately from local considerations; (ii) follows from Chow's theorem; (iii) follows immediately from standard results, cf. [3], [5]. The rest is obvious.

Proposition 4.1. Suppose that $\phi: Y \backslash\left\{\xi_{1}, \ldots, \xi_{r}\right\} \rightarrow \mathrm{R}^{n}$ is a complete, finitely branched minimal immersion which induces a branched metric of nonzero finite total curvature. Moreover, suppose that $\gamma_{\phi}$ takes values on a smooth curve $\mathscr{G} \subset Q_{n-2}$. Then:

(i) $\Gamma_{\phi}$ extends to give a holomorphic map $\Gamma_{\phi}: Y \longrightarrow \mathrm{P}(L \mathscr{G} \oplus \mathcal{O})$.

(ii) $\hat{Y}=\Gamma_{\phi}(Y)$ is an algebraic curve on $\mathrm{P}\left(L_{\mathscr{G}} \oplus \mathcal{O}\right)$.

(iii) $C(\phi)=-2 \pi(\hat{Y} \cdot C) \operatorname{deg}(\mathscr{G})$.

(iv) $\hat{Y} \cdot \mathscr{G}_{0}$ equals the total branching in the metric induced by $\phi$, counted with multiplicity.

(v) $\Gamma_{\phi}\left(B_{\gamma_{\phi}}\right) \subset \mathscr{G}_{\infty}$. Every other point in $\hat{Y} \cap \mathscr{G}_{\infty}$ corresponds to an end of the minimal surface.

REMARKS. (i) In (v), there are possibly both finite and infinite branch points of the Gauss map; the latter will give ends with 'spin', cf. [1].

(ii) For an algebraic curve on $\mathrm{P}(L \mathscr{G} \oplus \mathcal{O})$, other than $\mathscr{G}_{\infty}$, there is a finitely punctured part in $L_{\mathscr{G}}$. Integrating $\Omega \mathscr{G}$ over that part, for non-fibres, and taking the real component will give a minimal surface of the type described in 3.1, provided any periods are purely imaginary, cf. [1].

(iii) If $\mathscr{G}$ is a rational normal curve in $\mathrm{P}_{n-1}$, then $\mathscr{G} \cong \mathrm{P}_{1}$ and $\mathrm{P}(L \mathscr{G} \oplus \mathscr{O})$ is isomorphic to the rational normal scroll that has a curve of self-intersection number $-(n+1)$, cf. [2]. On this surface there is the linear equivalence $\mathscr{G}_{0} \sim$ $\mathscr{G}_{\infty}-(n+1) C$. In view of the existence of simple analogues of the Weierstrass 
formula for such surfaces, [7], it appears to be straightforward in this case to define the analogue of 'total spin at infinity' and obtain analogues of 3.12 in [1].

\section{Remarks on Another Formulation}

Proposition 4.1 is attractive but requires the image of the Gauss map to be smooth to be so formulated. We could formulate things over the desingularisation of the image but if that is necessary we might as well use the domain $Y$, i.e. pull the data back over $Y$. Thus, to $\phi: Y \backslash\left\{\xi_{1}, \ldots, \xi_{r}\right\} \longrightarrow \mathrm{R}^{n}$, as in 4.1 , there is associated a global mermorphic section $\eta \in \mathscr{M}\left(\left.T^{* *} Y \otimes \gamma_{\phi}^{*} J\right|_{Q_{n-2}}\right)$. Notice that there is a canonical $\mathrm{C}^{n}$-valued 1-form on the total space of $\left.T^{\prime *} Y \otimes \gamma_{\phi}^{*} J\right|_{Q_{n-2}}$, analogous to $\Omega$ above.

The number of zeros and poles, counted with multiplicity, of a meromorphic section $\eta$, is related to the degree of the line bundle through the classical formula:

$$
(\eta)_{0}-(\eta)_{\infty}=\operatorname{deg}\left(\left.T^{* *} Y \otimes \gamma_{\phi}^{*} J\right|_{Q_{n-2}}\right) .
$$

This leads immediately to a well-known refinement, which takes into account both the 'end multiplicity' and branch points structure, of the Chern-Osserman inequality: $C(\phi) \leq 2 \pi(\chi(Y)-2 r)$, cf. [4], [5].

The point here is that in Duistermaat's approach one considers $T^{* *} Y \otimes$ $\left.\gamma_{\phi}^{*} J\right|_{Q_{n-2}}$, a line bundle over $Y$.

\section{REFERENCES}

1. Duistermaat, J. J., Second Order Contact of Minimal Surfaces, Indag. Math. (N.S.) 13(3) (2002), 313-350.

2. Griffiths, P., and Harris, J., Algebraic Geometry, John Wiley, 1978.

3. Hoffman, D. A., and Osserman, R., The Geometry of the Generalized Gauss Map, Mem. Amer. Math. Soc. 28 No. 236, 1980.

4. Jorge, L. P., and Meeks, W. H., The Topology of complete minimal surfaces of finite total Gaussian curvature, Topology 22 (1983), 203-221.

5. Lawson, H. B., Lectures on Minimal Submanifolds, Vol I, Second Edition, Mathematics Lecture Notes Series 9, Publish or Perish, Inc., Wilmington, Del., 1980.

6. Osserman, R., A Survey of Minimal Surfaces, Dover Publications, Inc., New York, 1986.

7. Small, A., Duality for a class of minimal surfaces in $\mathrm{R}^{n+1}$, Tôhoku Math. J. 51 (1999), $585-601$.

MATHEMATICS DEPARTMENT

NATIONAL UNIVERSITY OF IRELAND, MAYNOOTH

CO. KILDARE

IRELAND

E-mail: asmall@maths.may.ie 